\title{
The Argo: A 65,536 channel recording system for high density neural recording in vivo
}

Kunal Sahasrabuddhe ${ }^{1}$, Aamir A. Khan ${ }^{1}$, Aditya P. Singh ${ }^{1}$, Tyler M. Stern ${ }^{1}$, Yeena Ng$^{1}$, Aleksandar Tadić ${ }^{1}$, Peter Orel ${ }^{1}$, Chris LaReau ${ }^{1}$, Daniel Pouzzner ${ }^{1}$, Kurtis Nishimura ${ }^{1}$, Kevin M. Boergens ${ }^{1}$, Sashank Shivakumar ${ }^{1}$, Matthew S. Hopper ${ }^{1}$, Bryan Kerr ${ }^{1}$, Mina-Elraheb S. Hanna ${ }^{1}$, Robert J. Edgington ${ }^{1}$, Ingrid McNamara ${ }^{1}$, Devin Fell ${ }^{1}$, Peng $\mathrm{Gao}^{2}$, Amir Babaie-Fishani², Sampsa Veijalainen $^{2}$, Alexander V. Klekachev², Alison M. Stuckey ${ }^{1}$, Bert Luyssaert², Takashi D. Y. Kozai ${ }^{3,4,5,6,7}$, Chong Xie ${ }^{8,9,10}$, Vikash Gilja ${ }^{11}$, Bart Dierickx², Yifan Kong1, Malgorzata Straka1, Harbaljit S. Sohal ${ }^{1}$, Matthew R. Angle ${ }^{1}$

\section{Author affiliations:}

1 Paradromics, Inc, Austin, TX, USA

2 Caeleste CVBA, Mechelen, Belgium

3 Department of Bioengineering, University of Pittsburgh, Pittsburgh, PA, USA

4 Center for the Neural Basis of Cognition, University of Pittsburgh, Pittsburgh, PA, USA

5 McGowan Institute of Regenerative Medicine, University of Pittsburgh, Pittsburgh, PA, USA

6 Center for Neuroscience, University of Pittsburgh, Pittsburgh, PA, USA

7 NeuroTech Center, University of Pittsburgh Brain Institute, Pittsburgh, PA, USA

8 Department of Electrical and Computer Engineering, Rice University, Houston, TX, USA

9 Department of Bioengineering, Rice University, Houston, TX, USA

10 NeuroEngineering Initiative, Rice University, Houston, TX, USA

11 Department of Electrical and Computer Engineering, University of California, San Diego, La Jolla, CA, USA 


\section{Abstract:}

Here we demonstrate the Argo System, a massively parallel neural recording system based on platinum-iridium microwire electrode arrays bonded to a CMOS voltage amplifier array. The Argo system is the highest channel count in vivo neural recording system built to date, supporting simultaneous recording from 65,536 channels, sampled at over $32 \mathrm{kHz}$ and 12-bit resolution. This system is designed for cortical recordings, compatible with both penetrating and surface microelectrodes. We have validated this system by recording spiking activity from 791 neurons in rats and cortical surface Local Field Potential (LFP) activity from over 30,000 channels in sheep. While currently adapted for head-fixed recording, the microwire-CMOS architecture is well suited for clinical translation. Thus, this demonstration helps pave the way for a future high data rate intracortical implant. 


\section{Introduction:}

Motor and sensory information are represented in the brain by coordinated ensembles of neurons with topographic maps that can span several centimeters in large animals and humans. Decoding information from these representations necessarily requires recording from large numbers of individual neurons with high temporal fidelity (Stevenson and Kording, 2011). As a result, there has been a recent impetus in both experimental and translational neuroscience to record from more neurons (Jun et al., 2017; Musk and Neuralink, 2019; Stringer et al., 2019; Obaid et al., 2020).

Within the field of experimental neuroscience, there have been significant advances in both electrical and optical methods for recording from large populations of neurons, each having its respective advantages. Optical methods for recording neural activity have made major strides through the development of fluorescent proteins such as genetically encoded calcium indicators (e.g. GCaMP6/7(Chen et al., 2013; Dana et al., 2019)) and voltage sensitive fluorescent proteins (e.g. Archon (Piatkevich et al., 2018) or QuasAR (Hochbaum et al., 2014; Piatkevich et al., 2018)). These new fluorescent probes enable functional imaging experiments that can simultaneously record from as many as 10,000 neurons in vivo (Hochbaum et al., 2014; Piatkevich et al., 2018; Stringer et al., 2019). While these are powerful experimental tools, approaches based on fluorescent proteins face significant barriers in clinical translation and can only record from shallow regions of the brain without implantable optics. Further, the expression of exogenous fluorescent proteins requires modification of host cells, which has substantial safety and regulatory implications when applied to humans. Lastly, the scattering of light in the brain and thermal sensitivity of brain tissue creates significant engineering challenges for developing a practically implantable imaging system that can spatially resolve activity without overheating tissue (Kozai and Vazquez, 2015; Acker et al., 2016). 
By contrast, electrical recording is well established as a tool for basic science and clinical research (Strumwasser, 1958). Intracortical electrode arrays are used for brain-computer interface applications (Hughes et al., no date; Hochberg et al., 2012; Flesher et al., 2016; Lubin, Strebe and Kuo, 2017; Pandarinath et al., 2017) and intraoperative recording (Truccolo et al., 2011; Misra et al., 2014; Cash and Hochberg, 2015). The state of the art in clinical neurophysiology, the Utah Array (Rousche and Normann, 1998; Hochberg et al., 2012), Blackrock Microsystems, USA), has enabled several notable applications in neural prosthetics (Willett et al., no date; Flesher et al., 2016; Pandarinath et al., 2017), despite having a limited electrode count (100 electrodes) and covering only a small $4 \mathrm{~mm} \times 4 \mathrm{~mm}$ cortical area. Success with the Utah Array has motivated efforts to record from more neurons over larger areas of the brain. The main approach has been the insertion of multiple Utah arrays in a single patient (Hughes et al., no date; Flesher et al., 2016), but the low density of electrodes and lack of multiplexing in the Utah array make the technology difficult to scale to higher channel counts.

Many recent efforts to 'scale up' neurophysiology have focused on lithographically patterned, thin film-based probes. Most notable are those based on active CMOS probes (e.g. Neuropixel (Jun et al., 2017)) and flexible thin-films stacks (e.g. Polyimide + metal (Chung et al., 2019; Musk and Neuralink, 2019)). These high channel-count devices have enabled novel experimental paradigms with acute and semi-chronic recording, but they have yet to be demonstrated as robust for chronic implantation in large animals. Thin silicon probes are fragile, and polymer-substrate probes commonly suffer from cracking (Kozai et al., 2015) and delamination between insulation and electrode (Ceyssens and Puers, 2015; Čvančara et al., 2020).

One technological approach that is both highly scalable and promises more immediate clinical application is the use of microwire-CMOS arrays (Kollo et al., no date; Obaid et al., 2020). These devices use arrays of parallel microwire electrodes, which are connected to active CMOS electronics for readout and stimulation. Microwire electrodes consist of a conductive metal wire 
core insulated by a solution-resistant dielectric such as a polymer or ceramic. Microwires have been used consistently and reliably over the last 70 years to record extracellular action potentials from the brains of experimental animal models and humans (Nicolelis et al., 2003; Jackson and Fetz, 2007; McMahon et al., 2014; Schwarz et al., 2014). They are highly robust and suitable for chronic applications (Nicolelis et al., 2003; Jackson and Fetz, 2007; McMahon et al., 2014; Schwarz et al., 2014) and translational models (Hubel, 1959; Hubel and Wiesel, 1959; Bartels et al., 2008; Misra et al., 2014). Importantly, recent work with carbon-fiber based probes suggests that the foreign body response to inserted microwires can be dramatically reduced by using wires with diameters less than $20 \mu \mathrm{m}$ (Kozai et al., 2012; Guitchounts et al., 2013; Patel et al., 2015).

Traditionally, microwire technology has been less scalable than integrated silicon probes due to the difficulty of connecting large arrays to a large number of amplifiers (Nicolelis et al., 2003; Schwarz et al., 2014; Sohal et al., 2014). Recently, however, simultaneous, parallel bonding of microwire arrays to high density CMOS sensor arrays have been successfully demonstrated (Obaid et al., 2020), paving the way for large format arrays of many thousands of wires that would not otherwise be achievable.

To date, demonstrations of the microwire-CMOS concept have involved adapting CMOS arrays that were not specifically designed for the application and have limited channel count due to restrictions in the array readout (Obaid et al., 2020). To fully realize the potential of microwireCMOS technology, we have designed a custom CMOS application-specific integrated circuit (ASIC), electronics, firmware, and software, providing an end-to-end solution for large scale recording.

This "Argo system" represents a significant advance in total data throughput, simultaneously addressing up to 65,536 channels at $32 \mathrm{kHz}$ and 12 bits of resolution. The CMOS readout array can be bonded to microwire electrode arrays of varying electrode length, count, and spacing, creating a highly versatile system applicable to different experimental models. It is designed for recording in a head-fixed, in vivo preparation. Full sensor readout produces a data 
rate of up to $26 \mathrm{Gbps}$, which can be simultaneously streamed directly to disk and viewed in realtime through a web browser-based digital oscilloscope interface. To validate our system, we performed initial recordings in the rat cortex with arrays of up to 1300 penetrating microwires, detecting action potentials from 791 single-units. To demonstrate the large channel count recording capability of the device, we performed surface recording of stimulus-evoked local field potentials from the sheep auditory cortex with a microwire-based electrocorticography array with over 30,000 channels.

\section{Methods:}

\section{System overview}

The Argo system is designed to enable simultaneous data acquisition on up to 65,536 channels at sampling rates of $32 \mathrm{kHz}$ (Khan et al., 2018). The system consists of an array of platinum-iridium (PtIr) microwire electrodes, a custom CMOS voltage-amplifier array designed to read and amplify neural signals (Kollo et al., no date; Obaid et al., 2020), electronics to process and packetize these signals, and a computer that runs the custom data acquisition software and user interface server (Figure 1). Individual components are described in detail in subsequent sections. 


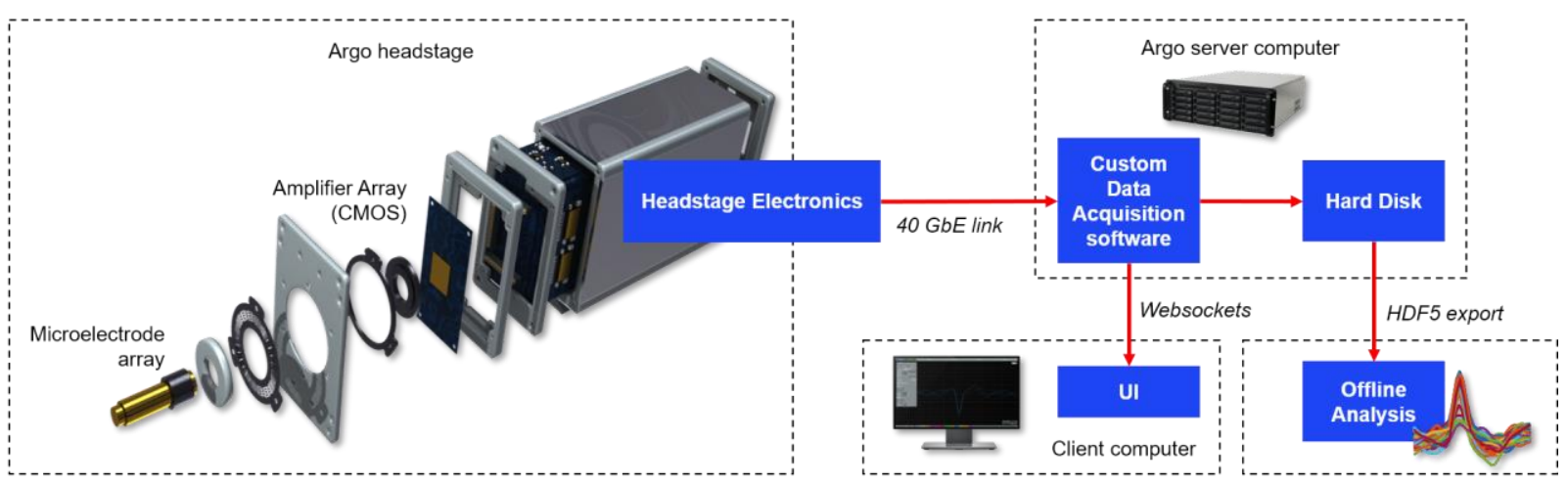

Figure 1: Block diagram of the Argo System. The Argo headstage comprises recording electrodes, the CMOS amplifier array, and electronics to digitize, packetize, and transmit signals over the optical data link. This data link connects it to the recording server computer, which hosts custom data acquisition software and a User Interface server. A client computer is used to read data from the server and display it in the system user interface. Data are exported as HDF5 files from the server and read into an offline processing computer where analysis and spike sorting are performed.

\section{Microwire Arrays for Neural Recording}

The recording array consists of a loosely ordered array of microwires (Tanaka Kikinzoku International, Japan) compressively and reversibly bonded to a custom CMOS amplifier array. The microwire cores are platinum-iridium alloy $(90 \% \mathrm{Pt} / 10 \% \mathrm{Ir})$, selected for their biocompatibility and demonstrated recording/stimulation performance in vivo (Geddes and Roeder, 2003; Cogan, 2008).

The fabrication process for our microwire arrays has been described previously (Obaid et al., 2020). For intracortical arrays, the distal ends of the microwire electrodes are electrosharpened in a parallel process adapted from (Musselman and Russell, 1990; Chang et al., 2012; Obaid et al., 2020). Microwires were bonded to a printed circuit board used to short all microwires and deliver electrical signals to them, and their distal ends were dipped in a $0.5 \mathrm{M} \mathrm{CaCl}_{2}$ solution. Sharp tips were formed by controlling the voltage applied to the wires in solution, and the speed at which wires were drawn out of solution. The amplitude, frequency, pulse duration, and duty cycle of the voltage applied to the wires were modulated using a Chroma Programmable AC 
Source (Model 61603, Chroma ATE, Taiwan). The wire speed was controlled using a stepper motor (ZST225B, ThorLabs, USA) and stepper motor controller (KST1, ThorLabs, USA), wires could be sharpened to arbitrary taper lengths and tip angles. The wire sharpening process consisted of four steps: (1) wire length equalization, (2) coarse electrosharpening, (3) fine electrosharpening, and (4) electrochemical polishing of the wire tips.(See Supplementary Table S1 for system parameters, and Supplementary Table S2 for sharpening process parameters). Between steps, the solution is circulated through a large bath using a preristaltic pump (Model 07528-10, Cole Parmer, USA) to evacuate reaction products. The final tip diameter is smaller than $200 \mathrm{~nm}$ (Figure 2A) to alleviate the "bed-of-nails", or dimpling, effect that is commonly associated with the implantation of high density electrode arrays ( $<400 \mu \mathrm{m}$ pitch) into cortical tissue (Obaid et al., no date; Nordhausen et al., 1992). Non-penetrating electrode arrays do not require this sharpening step, therefore tip preparation is performed later in the process.

Microwires are then insulated with a thick sacrificial parylene-C layer to set the electrode spacing $(100-400 \mu \mathrm{m})$. Following the coating step, the wires are tightly packed into a fluorinated ethylene propylene (FEP) heat shrink tube that is then shrunk to fix the wire positions within the array. Next, the proximal end of the array is prepared to ensure good electrical contact with the CMOS array when the array is mechanically pressed against the face of the sensor array (Obaid et al., 2020). This is achieved by polishing the proximal end of the array to be flat and then exposing the microwires at the proximal ends $10-20 \mu \mathrm{m}$ by ashing in oxygen plasma using an SPI Plasma Prep III system (SPI Supplies, USA).

For non-penetrating electrode arrays, the tips are prepared by polishing the entire distal end of the array at this stage. This ensures that the recording sites are co-planar, smooth, and appropriately exposed.

For penetrating electrodes, the shank lengths of the distal ends (tips) of the bundled microwires are defined by ashing them with oxygen plasma as described above. At this stage, the arrays are coated with a robust $200-300 \mathrm{~nm}$ Atomic Layer-Deposited (ALD) alumina coating 
(Savannah S200, Cambridge NanoTech, MA, USA) to provide a high-quality insulation layer (Xie et al., 2014) that is then selectively de-insulated to define the length of the recording site at the wire tip.

Recording sites have a typical impedance of around $300-500 \mathrm{k} \Omega$ at $1 \mathrm{kHz}$ in physiological saline. The smaller arrays used for in vivo experiments in rats had between 100 and 1300 recording electrodes, while the larger arrays prepared with flat tips for Local Field Potential (LFP) recordings in sheep had over 30,000 electrodes. 

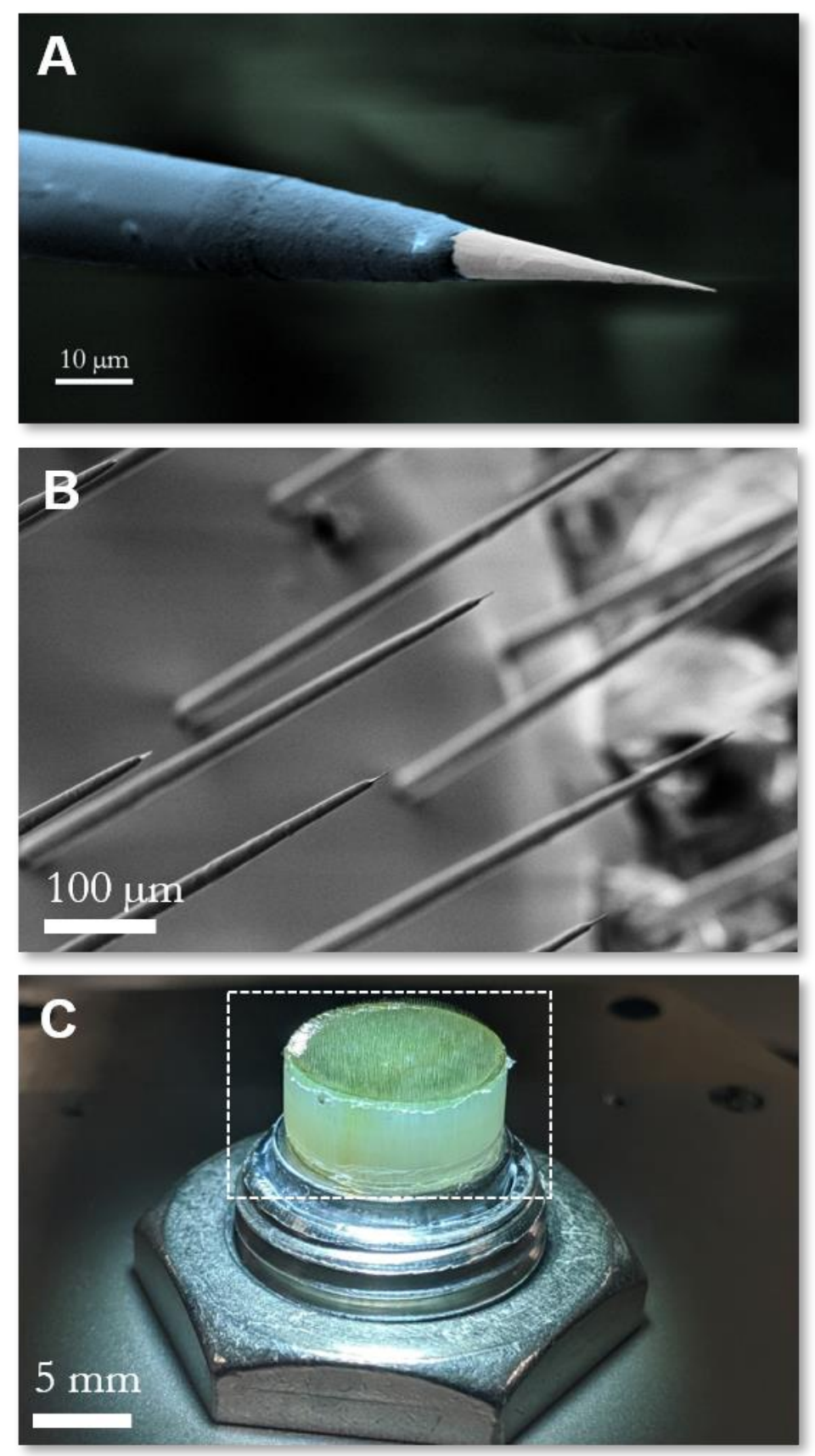

Figure 2: Microwire recording electrodes. (A) False color electron micrograph of a single electrosharpened microwire showing taper along the electrode length, alumina insulation layer (blue) and recording site at the tip (white), and (B) micrograph of the distal end of the microwire array showing a field of electrodes with electrosharpened tips. (C) Macroscopic optical image of the electrode array, highlighting the etched back recording end (white dashed box). 


\section{CMOS Sensor Design}

The sensor in the Argo system is an application-specific integrated circuit (ASIC) designed to amplify and filter neural signals from a high-density microwire array. It was codesigned by Paradromics and Caeleste, CVBA (Belgium) and fabricated by X-FAB Silicon Foundries (Germany and Malaysia) in a $180 \mathrm{~nm}$ CMOS process technology node. The sensor consists of a pixel array of $256 \times 256$ pixels with a pixel pitch and dimensions of $50 \mu \mathrm{m} \times 50 \mu \mathrm{m}$, adding up to an active area of $12.8 \mathrm{~mm} \times 12.8 \mathrm{~mm}$ for the readout array. The peripheral circuit elements for control and readout increase the total dimensions of the ASIC to $14.5 \mathrm{~mm} \times 16 \mathrm{~mm}$.

Each pixel has a $40 \mu \mathrm{m} \times 40 \mu \mathrm{m}$ top metal pad which is used as the landing pad for the microwire electrode. This top metal pad is AC-coupled to a low-noise amplifier (LNA) chain. The LNA chain is composed of three main blocks: input amplifiers, an antialiasing low-pass filter, and an output column buffer (Figure 3).

The amplifiers are implemented as common-source gain stages that are biased to operate in the class A regime. This topology was chosen due to its good linear response, high input impedance, and low noise. The drawbacks are higher power consumption and lower gain. To limit the power consumption and at the same time achieve the required gain of $100 \mathrm{~V} / \mathrm{V}$, the design implements two amplifiers in series, with each amplifier contributing a gain of approximately $10 \mathrm{~V} / \mathrm{V}$. In addition, each amplifier has a tunable input biasing circuit which in conjunction with an $A C$ coupling input capacitor forms a tunable high-pass filter. Thus, the amplifier block provides a cascaded second order high-pass filter that has its $3 \mathrm{~dB}$ point tunable from approximately $18 \mathrm{~Hz}$ to $300 \mathrm{~Hz}$. This serves two functions. The first is the removal of DC offsets, drifts, and slowly varying out-of-band high amplitude signals. These undesired signal components can saturate the input and diminish the signal dynamic range, ultimately reducing the ability of the system to acquire meaningful signals. The second is to provide the flexibility of the system to operate in two modes. In one mode, setting the corner frequency to $18 \mathrm{~Hz}$ enables both neuron spiking activity and LFP to be recorded, since the latter has most of its integrated 
power below $100 \mathrm{~Hz}$. In the second mode, LFP signals can be rejected by increasing the corner to higher frequencies. This allows for spiking signals to retain the entire dynamic range. Furthermore, the input impedance is sufficiently high as to not form a significant voltage divider with the otherwise high electrode impedance, which can range as high as $10 \mathrm{M} \Omega$ at $1 \mathrm{KHz}$. Specifically, the input impedance at $1 \mathrm{kHz}$ has a resistive component of $4.4 \mathrm{G} \Omega$ and a capacitive component of $0.4 \mathrm{G} \Omega(400 \mathrm{fF})$.

To keep the integrated noise floor as low as possible while simultaneously providing antialiasing, the amplifier chain is followed by a third order low-pass filter with a tunable corner frequency between $8 \mathrm{kHz}$ and $50 \mathrm{kHz}$. In our application all three corners are set to $20 \mathrm{kHz}$ resulting in a $3 \mathrm{~dB}$ point at approximately $12 \mathrm{kHz}$ and providing good signal suppression at the Nyquist frequency of $16 \mathrm{kHz}$.

The output buffer of the pixel provides isolation between the pixel output and the column line used to multiplex the pixels in a single column. That is, when one of the pixels in the column is being read out the other pixels have their outputs disconnected from the line to avoid overloading. This reduces crosstalk between pixels and ensures that each pixel can read out signals from unique electrodes.

In the ASIC periphery, a set of control circuits and high-bandwidth amplifiers convert the multiplexed signal from single-ended to differential and bring the total gain of the signal chain to around $800 \mathrm{~V} / \mathrm{V}$. The sensor multiplexes 2048 individual channels to each of 32 high-speed analog outputs from the entire array. Furthermore, the output buffers are designed to drive long transmission lines leading to the external analog-to-digital converters ADCs. 


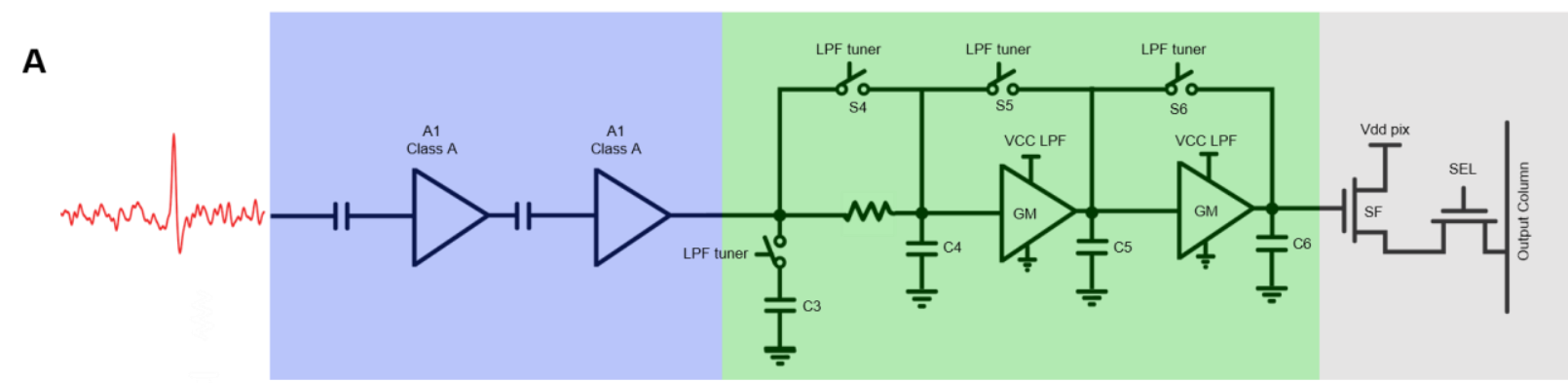

Figure 3: CMOS sensor characterization. The input signal is AC-coupled into two source followers biased in the Class A configuration (A1, A2, shown in blue), which together form the front-end low noise amplifier (LNA) chain. Each stage has a gain of $10 \mathrm{~V} / \mathrm{V}$. The third stage (green) is a third-order tunable low-pass filter that serves as an anti-aliasing filter. The final stages (grey) are for pixel selection to read out the stored value.

\section{Argo System Electronics}

The system electronics consist of two custom printed circuit boards (PCBs). The first PCB (frontend board) is designed to house the CMOS sensor and support electronics. The second PCB (main board) is designed to digitize analog signals and deliver them to the server (Figure 4). It is designed in a rigid-flex form factor for the reasons of signal integrity, compactness, and ease of assembly.

The CMOS sensor is wire-bonded to the front-end board, which also contains a reference connector and a set of precision voltage regulators for the sensor. The front-end board is mounted in a metal housing designed to reliably connect with the microwire array after pressing (see Methods: Array connectivity testing for more details) (Kollo et al., no date).

The main board contains two 16-channel high-speed ADCs (ADS52J90, Texas Instruments, USA) as well as a Field-Programmable Gate Array (FPGA, XC7K160T-2FFG6762, Xilinx, USA). The headstage requires an external 5V/3A DC power source and is kept galvanically isolated from powerline and other electronic noise sources, which can otherwise corrupt neural signals. The main board is housed within a separate headstage body designed to connect with the front-end main board frame. Electrical connections between the two boards are 
made via high-density spring-loaded pins, shown in Figure 4(D)) (855-22-040-30-001101, MillMax, USA). This allows reliable and repeatable mating between the boards without imposing extreme tolerance constraints on the fabrication of either the boards or the mechanical components. Two banks of pins transmit the multiplexed, high-speed analog output signals from the CMOS sensor to the ADCs (Figure 4(E)), while the third separate bank of pins transmits control signals from the FPGA to the CMOS sensor.

The ADC's input signal range of $2 \mathrm{Vpp}$ translates to an input signal range of $\sim 2.5 \mathrm{mVpp}$ in the CMOS amplifiers, given the 800 V/V gain (see Methods: CMOS Sensor Design). The ADCs operate at a sampling frequency of $78.125 \mathrm{MHz}$, corresponding to a sensor sampling rate of $32 \mathrm{kHz}$, and digitize the analog signal to a 12-bit digital signal that is then transmitted to the FPGA over tightly impedance-controlled Low-Voltage Differential Signalling (LVDS) pairs. This high sampling rate provides further protection from aliasing artifacts. It also allows for spike sorting applications of the neural data, wherein a higher sampling rate leads to better cluster/unit separability in PCA space for an individual spiking channel (Lewicki, 1998; Ghanbari, Papamichalis and Spence, 2009).

The FPGA (Figure 4(G)) then demultiplexes the wide-band digitized signals from the 32 LVDS channels into the original 65,536 channels and remaps them to construct a $256 \mathrm{x}$ 256pixel raster at $32 \mathrm{kHz}$. The logic in the FPGA generates timing signals for raster scanning the $256 \times 256$ pixels CMOS sensor array at a rate of up to 32,000 frames per second.

The FPGA is also used to coordinate data transfer to the host computer via a 40 gigabit Ethernet link through an OM3 optical fiber (943-99684-10005, Amphenol, USA). As the physical layer of the 40GBASE-SR4 protocol, the FPGA drives a Quad Small Form-factor Pluggable (QSFP+) optical transceiver (Figure $4(\mathrm{H})$ ) through its 8 GTX transceivers. An embedded application processor (Xilinx Microblaze) within the FPGA fabric coordinates the ARP, ICMP, and UDP communication for control signals to and from the host computer. In order to maintain 
throughput for the high-speed neural data, the FPGA fabric directly assembles UDP packets using header information set by the embedded processor.

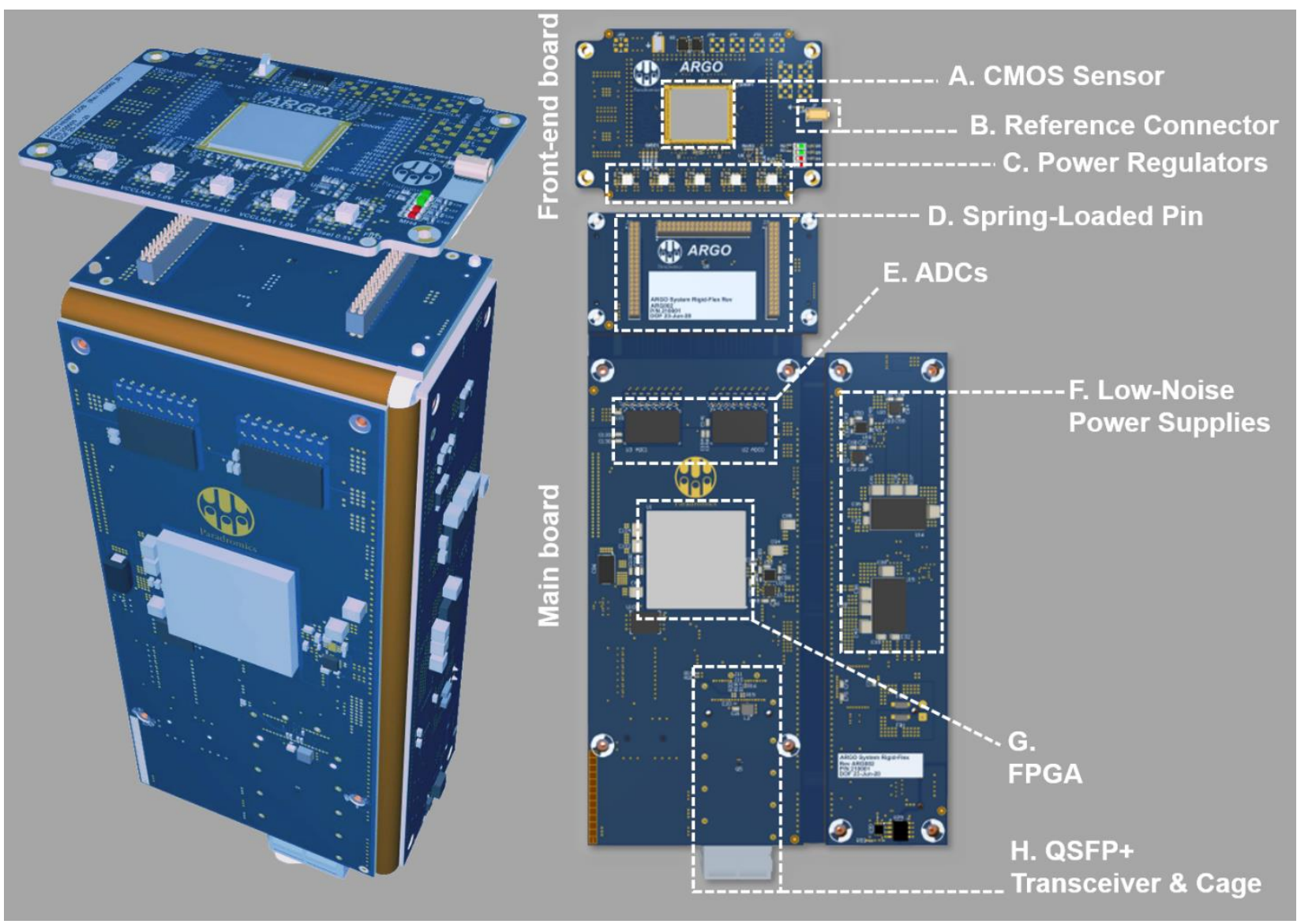

Figure 4: System electronics. The Argo system's electronics are housed on two custom PCBs. (Left) PCBs are folded and aligned to fit the metal housing of the Argo system. (Right) A smaller PCB (the front-end board) holds the CMOS sensor $(A)$, reference connector $(B)$, and power regulators $(C)$ for the sensor. This front-end board is connected to a larger main board via three banks of spring-loaded connectors (D). On this main board sit the analog-to-digital converters (ADCs, E), low-noise power supplies (F), the FPGA (G), and the Quad Small Formfactor Pluggable (QSFP+) transceiver and connector $(\mathrm{H})$ over which data are transmitted using an optical fiber (not pictured). 


\section{Computer systems for neural readout and data storage}

To handle the large amount of data generated by the Argo system, the host computer is custom-built on a Supermicro dual Xeon server platform (X10DAC, Supermicro, USA) running Ubuntu Linux 18.04 Server Edition and equipped with two Intel Xeon E5-2640 v4 processors, 64 GB of ECC RAM and an Intel XL710 network interface card for 40GBASE-SR4 communication with the Argo headstage. This host server runs a fully custom data acquisition software package. This software produces two data streams: one raw data stream to be written to disk, and another stream to be served externally through a websockets interface, where data can be accessed through the application programming interface (API). The data acquisition software package is built on Intel's Data Plane Development Kit (DPDK) to ingest the raw ethernet packets at full 40 Gbps line rate. The packets are evaluated for checksum integrity at the application level and are rearranged to be written to an array of 20 hard drives using Direct Memory Access (DMA) and a custom multi-threaded sharding system.

High-performance computing technologies such as core pinning, cache line optimization, and memory alignment are used throughout the data acquisition software stack to enable recording of full frame neural data at full $32 \mathrm{kHz}$ frame rate. This allows the user to record data continuously for up to 8 hours, the duration of a typical acute sheep experiment, without running out of disk space or needing multiple recording systems in the surgical environment.

\section{User interface for live data visualization}

A custom UI for real-time monitoring was developed for the Argo system to facilitate inexperiment data validation required for collection of high-quality datasets. Some salient features of the UI include customizable bandpass filters (e.g. 300-6000 Hz for recording in the spike band), powerline filters (50/60 Hz), common average referencing (Ludwig et al., 2009) across all or a selected subset of channels and the ability to listen to channels of interest. These 
features were used to ensure that denoising was performed appropriately prior to the experiment and to determine whether data collected was electrophysiological in nature.

By default, the $\mathrm{UI}$ is initialized with a raster image of the sensor, producing a spatial map of activity on all channels. This enables the user to implement connectivity masks (see Methods: Array connectivity testing for more detail). Each pixel on the raster represents an individual amplifier channel, and each channel's raw or filtered output can be viewed in a separate tracescope, in either live-stream mode, or in threshold-trigger mode, similar to an oscilloscope.

The UI server also has an API that can be used to control the system through an external program such as MATLAB to facilitate additional analyses (e.g. channel RMS values or power spectrum plots) during recording sessions.

\section{Array connectivity testing}

To complete the assembly of the device, microelectrode arrays were physically pressed onto the chip while monitoring connectivity. The connectivity was assessed in the physiological saline bath by submerging electrode recording sites while applying a $2 \mathrm{kHz},>2 \mathrm{mV}$ PP sine wave. Using the UI described previously, the pressed array was adjusted until maximal connectivity was observed and the press was stabilized with set screws on the housing within the front-end board. This completed the process of bonding the microwire electrode array to the CMOS sensor.

Next, a quantitative connectivity test was performed to determine the gain and noise of the electrically coupled array-chip device. Gain and noise were calculated using a custom MATLAB program (MATLAB 2018a, Mathworks, USA) that fetches data through the system API described previously. The gain on every pixel was calculated by applying a $2 \mathrm{kHz}, 0.5 \mathrm{mV}$ peak to peak sine wave signal in the saline bath and measuring the recorded voltage. The noise was then calculated by shorting the system reference to the saline bath (with no external signal applied). The noise measurement was band-pass filtered between $300 \mathrm{~Hz}$ and $6 \mathrm{kHz}$ (i.e., within 
the spiking band). The input-referred noise was determined using the recorded root mean squared (RMS) noise values and the gain for each pixel calculated in the previous step.

The connectivity map was determined using $k$-means clustering of gain and noise values. Clusters with appropriate gain (mean $>650 \mathrm{~V} / \mathrm{V}$ ) and noise (mean $<10 \mu \mathrm{V}_{\mathrm{RMS}}$ ) were considered optimally connected to the system, with the number of clusters set interactively by the user to achieve these thresholds. From this analysis, a connectivity mask was generated to remove unconnected channels from subsequent analysis.

\section{Animal Surgery}

\section{Rat Surgery}

This study and all experimental protocols were approved by the Institutional Animal Care and Use Committee (IACUC) at the University of Texas at Austin, which follows the National Institutes of Health $(\mathrm{NIH})$ guidelines for the ethical treatment of animals. Male Sprague Dawley rats (Charles River, 250-400 g) were used for implanting Argo microwire arrays. Rodents were housed in a laboratory environment on a 12-h light and dark cycle at $25^{\circ} \mathrm{C}$, with ad libitum access to food and water.

Animals were anesthetized in an induction chamber on $3 \%$ isoflurane and then transferred to a small animal stereotaxic frame (Narishige, Japan) and maintained at $2.5 \%$ isoflurane for the duration of the procedure. Hair was removed from the head with small animal shears, Puralube (Dechra, UK) was placed on the eyes, and the surgical sites were sterilized with alternating povidone-iodine and alcohol pads prior to incision. Temperature was monitored rectally, and a heat mat was placed under the animal for the duration of the procedure with temperature maintained at around $36.0-36.5^{\circ} \mathrm{C}$.

An incision was made through the scalp exposing the skull. The fascia was reflected and the periosteum was removed from the bone. A small craniotomy was made $(5 \times 5 \mathrm{~mm})$ with a 
microdrill (OmniDrill 35, World Precision Instruments, USA) and the margins were removed with rat skull-appropriate bone rongeurs to extend the craniotomy to accommodate a $10 \mathrm{~mm}$ diameter microwire array. A durotomy was then performed with the use of microscissors. Once the durotomy was completed a micromanipulator was used to insert the Argo Microwire array into the brain and physiological saline was applied to keep the brain moist during the experiment. Argo microwires were inserted $0.7-1 \mathrm{~mm}$ into the brain to ensure that most of the electrodes would reside in the grey matter of the brain to enable recording of neural unit activity.

To create a reference electrode, an $80 \mu \mathrm{m}$ diameter teflon (PTFE)-coated PtIr wire (AM Systems, USA) was de-insulated 1-2 $\mathrm{mm}$ from one end. The deinsulated end was then placed in the subdural space. The wire was secured in place with gel foam (Pfizer, USA) and secured on the skull with the application of UV-curable dental acrylic (Flowable Composite 101-6773, Henry Schein, USA). This limited the motion of the reference wire to reduce the possibility of picking up microphonic noise.

\section{Sheep Surgery}

This study and all experimental protocols were approved by the Institutional Animal Care and Use Committee (IACUC) at the Bridge PTS, San Antonio, Texas, which follows the National Institute of Health (NIH) guidelines for the ethical treatment of animals. White face Dorset Sheep (Ovis aries) that weighed 30 to $35 \mathrm{~kg}$ were used for this study. Food was withheld for 24 hours prior to surgery, while water was provided to the sheep ad libitum.

Sheep were induced by using Tiletamine/Zolazepam (Telazol, 4-6 mg/kg, IM). The sheep was intubated, and anesthesia was maintained via $1-5 \%$ isoflurane delivered in $60 \%$ oxygen and $40 \%$ medical grade air. An orogastric tube was placed to minimize or prevent ruminal bloat. Ophthalmic ointment (Paralube, Dechra, UK) was applied to prevent corneal desiccation. Thermal support was provided via a circulating warm water blanket (T/Pump, Stryker, USA) during the course of anesthesia or Bair hugger (3M, USA). 
Once the sterile field was prepared, an incision was made over the skull to expose the bone and underlying fascia. The tissue was reflected, and the periosteum removed over the exposed skull. Next, a surgical microdrill (OmniDrill 35, World Precision Instruments, USA) was used to perform the craniotomy. Bone rongeurs were used to remove excess bone and fully expose the surface of the dura. A craniotomy was made (typically $3 \times 3 \mathrm{~cm}$ ) over the auditory cortex in the sheep. Typical stereotaxic coordinates were $5 \mathrm{~mm}$ anterior and $25-30 \mathrm{~mm}$ medial from bregma point for the center point of the craniotomy. After the craniotomy, a durotomy was performed to expose the pia with the use of microscissors. The surface of the brain was kept moist with the aid of physiological saline soaked gel foam (Pfizer, USA) throughout the experimental procedure.

To create a reference electrode, an $80 \mu \mathrm{m}$ diameter teflon (PTFE)-coated PtIr wire (AM Systems, USA) was de-insulated 1-2 $\mathrm{mm}$ from one end. The deinsulated end was then placed in the subdural space. The wire was secured in place with gel foam (Pfizer, USA).

During the procedure, vitals were closely monitored (e.g. $\mathrm{SPO}_{2}$, respiratory rate). To minimize the effects of brain pulsation, end-tidal $\mathrm{CO}_{2}$ was typically maintained between $30-40$ $\mathrm{mmHg}$ using mechanical ventilation. At the conclusion of the experimental procedures, animals were euthanized with an overdose of sodium pentobarbital (110 mg/kg, IV).

\section{In-Vivo recordings}

\section{Electrophysiology system}

Electrophysiology recordings were acquired using the Paradromics Argo System at a sampling frequency of $32 \mathrm{kHz}$. Typically for the recording procedure, isoflurane was reduced to $<2 \%$ to reduce the effect of anesthesia on neural activity. 


\section{Rat Action Potential Recordings}

Microwire arrays between $5 \mathrm{~mm}$ and $10 \mathrm{~mm}$ in diameter $(\mathrm{n}=5)$ were implanted into the somatosensory and prefrontal regions of the rat cortex. These areas were targeted for the ease of insertion of the array into the rat cortex due to the limited brain size relative to the array diameter. A successfully inserted electrode typically had a peak-to-peak noise floor amplitude of $25-30 \mu \mathrm{V}$.

Spiking channels of interest were evaluated in real-time with the oscilloscope and strip chart modes on the UI to confirm the presence of spike-like waveforms (typical peak width $<1$ ms). We also examined specific channels of interest to observe neural firing patterns and ensure that high frequency noise was not corrupting the signal.

As a final confirmation of that the signals recorded were physiological in origin, isoflurane levels were often increased to $5 \%$ at the end of the procedure to observe the loss of neural activity and the return of the channels to the $25-30 \mu \mathrm{V}$ noise floor.

\section{Auditory Paradigms}

Auditory stimuli were presented free-field through multifield speakers (MF1, Tucker-Davis Technologies, USA) approximately $10 \mathrm{~cm}$ from the ear contralateral to the recording hemisphere. Signals were controlled using custom MATLAB software via a data acquisition system (USB6366, National Instruments, USA) at a sampling rate of $192 \mathrm{kHz}$, which delivered the signal through an amplifier (SA1, Tucker-Davis Technologies, USA). The speaker was calibrated using a 0.25 inch condenser microphone (PCB Piezotronics,USA).

Pink noise was presented at 80 dB Sound Pressure Level (SPL) (100 ms duration with $10 \mathrm{~ms}$ rise/fall $\cos ^{2}$ ramp), with an inter-stimulus interval of $600 \mathrm{~ms}$. For the rat, 800 stimuli were presented, and for the sheep, 500 stimuli were presented. 
For the sheep, pure tones were also presented (50 ms duration with $10 \mathrm{~ms}$ rise/fall $\cos ^{2}$ ramp) at levels from 40 to $80 \mathrm{~dB}$ SPL in $10 \mathrm{~dB}$ steps, at an inter-stimulus interval of $500 \mathrm{~ms}$. Pure tones ranged from 0.5 to $32 \mathrm{kHz}$ with 3 steps/octave, with 20 repetitions per stimulus.

\section{Offline Recording Analysis}

Exported HDF5 files were imported into MATLAB. Channels were bandpass filtered (using the built-in 'filtfilt' function, forward and backward filtering to eliminate phase delays and distortions) from 300-6000 Hz. Channels were visually inspected, and their power spectra plotted, to confirm no noise source contamination. After initial data checks, spike sorting was performed on all the channels.

Spike sorting was performed using Wave_Clus (Quiroga, Nadasdy and Ben-Shaul, 2004). Typical thresholds for neural data were set at least 3.5 times the noise threshold, calculated through Wave_Clus. In short, the noise threshold $(\sigma)$ was calculated by taking the mean of the absolute value of the bandpassed signal and dividing it by 0.6745 , and the spike crossing threshold was set at a minimum of 3.5 $\sigma$ (Quiroga, Nadasdy and Ben-Shaul, 2004). The output of the batch processing was then used to confirm the presence or absence of spike waveforms. Single units were confirmed by three metrics: (1) all neural waveforms had a peak width less than 1 ms. (2) A neural interspike interval histogram (Quiroga, Nadasdy and Ben-Shaul, 2004; Rossant et al., 2016) with a clear indication of a refractory period (i.e. no waveforms in the 0-3 ms bins on Wav_Clus output) was observed. (3) Clusters were clearly separated, as confirmed through the Wave_Clus user interface. Waveforms that did not match these criteria were deemed not to be single units and were not used for subsequent analysis. 


\section{Neural Activity Quality Metrics}

To evaluate the recording quality of our isolated single units we calculated the peak-topeak amplitude (P2P), noise, and signal-to-noise ratio (SNR). P2P was calculated by taking the peak-to-peak amplitude of median waveform of the sorted putative single unit. The noise (StdNoise) was then determined by calculating the standard deviation of the RMS noise of the band-passed signal and multiplying it by two (Kipke et al., 2003; Ludwig et al., 2011; Sohal et al., 2014). SNR was then calculated as P2P/StdNoise.

\section{Sheep LFP recordings}

\section{Signal Conditioning}

For LFP data analysis, all channels in the connectivity map generated immediately prior to the experiment were digitally band-pass filtered from $2 \mathrm{~Hz}$ to $400 \mathrm{~Hz}$, with additional filtering to remove $60 \mathrm{~Hz}$ harmonics, and the resulting data were decimated to an effective sampling frequency of $1 \mathrm{kHz}$. To highlight evoked responses to pure tones stimuli in particular, and to further increase SNR for these recordings, the data were denoised by applying independent component analysis (ICA) (Hyvärinen and Oja, 2000). The data were first scaled and whitened using principal component analysis, before performing ICA decomposition. Next, the data were further processed by applying the Hilbert transform and evoked responses were constructed from the envelope $(2-400 \mathrm{~Hz})$ of the signal amplitude to account for the oscillatory nature of the LFP waveforms.

\section{Pink Noise Response Analysis}

Evoked responses to pink noise were compared to baseline activity for each channel. The average baseline across trials was calculated using a time window of $100 \mathrm{~ms}$ preceding stimulus onset to stimulus onset, and the average response was determined using a window from $20 \mathrm{~ms}$ to $120 \mathrm{~ms}$ after stimulus onset. This additional $20 \mathrm{~ms}$ delay after stimulus onset was to 
accommodate for the minimum latency that we observed for auditory responses for surface LFP recordings, similar to that previously reported in other model systems (Kajikawa and Schroeder, 2011; Escabí et al., 2014; Trumpis et al., 2017). The $\Delta \mathrm{RMS}$ was calculated by subtracting the RMS of the baseline window from the RMS of the signal in the response window. Channels having a $\triangle \mathrm{RMS}$ of greater than $10 \mu \mathrm{V}$ were considered responding channels, and evoked responses for each channel were considered statistically significantly different from baseline activity if $p<0.05$, using a multiple comparisons permutation test (Nichols and Holmes, 2002).

\section{Tone Response Analysis}

Responses to each tone were averaged across trials and across all levels presented, since most responses were above threshold at the lowest presented level (i.e., $40 \mathrm{~dB}$ SPL). The non-parametric Wilcoxon Rank Sum tests with Bonferroni correction were used to distinguish statistically significant evoked responses from baseline ( $p<0.05 /$ number of channels), as the data were non-gaussian. A channel was considered responsive to pure tones if it had a statistically significant response to at least two tones (i.e., 0.6 octave bandwidth), and a $\Delta \mathrm{RMS}$ of at least $10 \mu \mathrm{V}$, which was considered the minimum response threshold. For these responding channels, the Best Frequency (BF) for each channel was determined as the tone which evoked the most significant response.

\section{Results:}

Here we designed and characterized the Argo system, which included a microwire electrode array of up to 31,000 channels bonded to a custom CMOS chip. Subsequent sections describe our characterization and validation of the system, which includes bench testing and performing in vivo experiments to record spikes in the rat cortex with 1300 microwires and surface LFP from the sheep auditory cortex with $>30,000$ microwires. 


\section{Benchtop characterization of the bonded microwire-electronics system}

We validated the system through bench testing physiological saline by evaluating the connectivity between the microwires electrodes and the amplifier inputs on the CMOS-sensor. Although electrodes at the periphery of the array occasionally showed lower connectivity, we were typically able to connect the majority of electrodes to an amplifier input using this process.

Connectivity tests were performed for every electrode array and sensor combination that was assembled. For 32 sensor/microwire array combinations, we obtained $71 \pm 2.9 \%$ connectivity(+/-SEM) for all electrode pitches tested $(60-300 \mu \mathrm{m})$.

The microelectrode array used for surface LFP recordings had an electrode pitch of 60 $\mu \mathrm{m}$ and the resulting $12 \mathrm{~mm} \times 12 \mathrm{~mm}$ array contained $\sim 35,000$ electrodes. For this array, 30,146 pixels were connected to microwire electrodes, giving $86 \%$ connectivity (Figure 5A). The gain (+/SD) was $811 \pm 21 \mathrm{~V} / \mathrm{V}$, and the corresponding input-referred noise (+/-SD) was $6.3 \pm 0.5 \mu \mathrm{V}_{\mathrm{RMS}}$ in the $300-6000 \mathrm{~Hz}$ band (Figure 5B). From our $k$-means gain and noise analysis, $99.2 \%$ of the connected channels had $<10 \mu \mathrm{V}_{\mathrm{RMS}}$ band-limited input-referred noise.

Across 32 CMOS sensor-bonded microwire arrays, the gain (+/- SD) was $763 \pm 7 \mathrm{~V} / \mathrm{V}$, and the corresponding input-referred noise (+/-SD) was $7.5 \pm 0.4 \mu \mathrm{V}_{\mathrm{RMS}}$ in the $300-6000 \mathrm{~Hz}$ band. Thus, a large array of functional electrodes can be created using this CMOS sensormicrowire bonding approach with high connectivity across the sensor and good gain/noise characteristics for connected channels. 

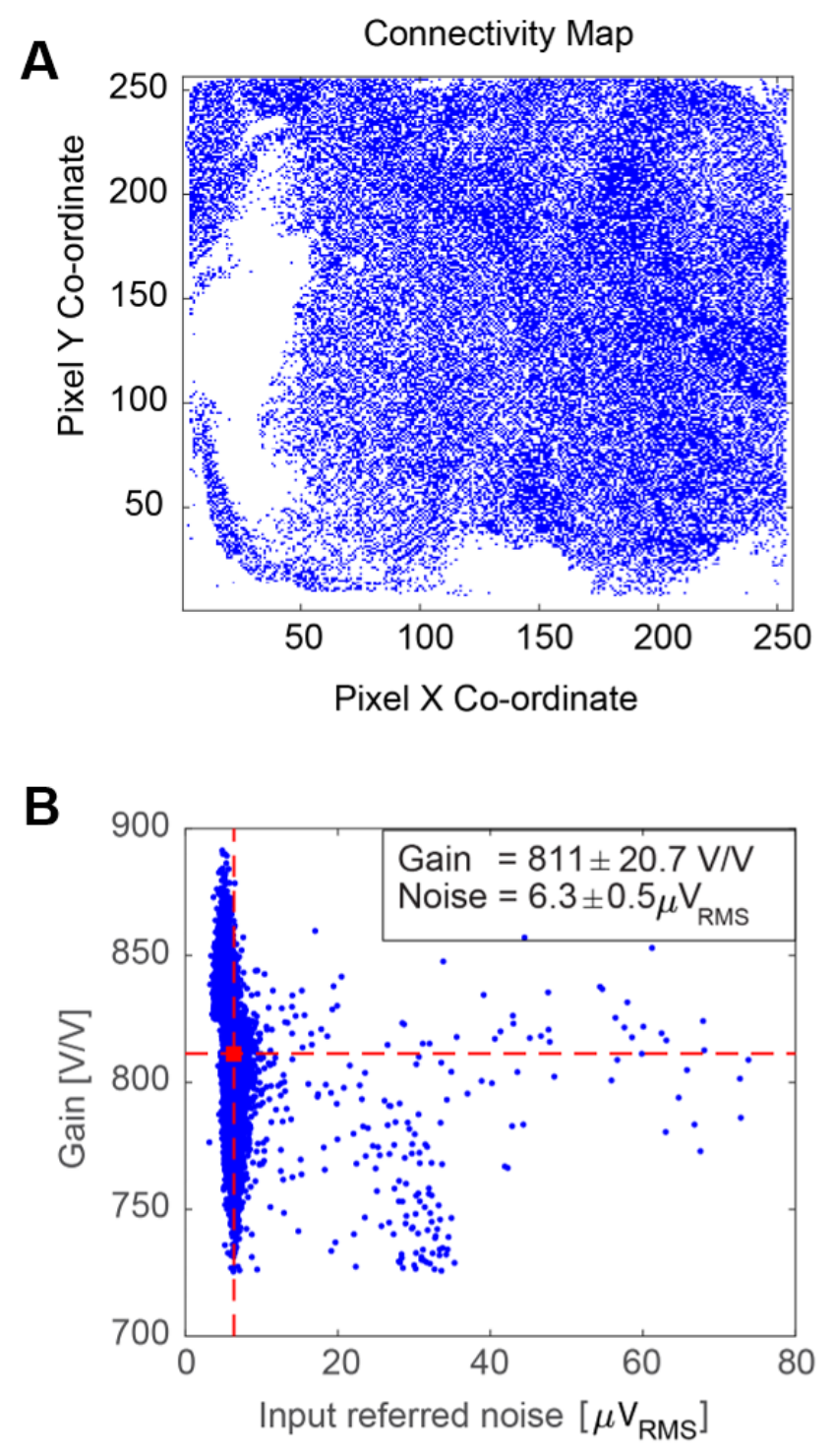

Figure 5: Full signal chain characterization in saline bath prior to in vivo experiment. (A) Raster image of the full sensor showing pixels connected to active electrodes for an array of approx. 35,000 electrodes. (B) Gain and noise distribution for the connected pixels. Channel clusters with mean gain $>650 \mathrm{~V} / \mathrm{V}$ and mean noise $<10 \mu \mathrm{V}_{\mathrm{RMS}}$ were selected for further analysis; here, the mean gain was $811 \mathrm{~V} / \mathrm{V}$ (horizontal dashed red line), and the mean noise was 6.5 $\mu \mathrm{V}_{\text {RMS }}$ (vertical dashed red line). The final connectivity map contained 30,146 channels for analysis, resulting in a connectivity of $86 \%$.

\section{Rat Cortical Recordings}

To validate our system in vivo, we show data from a 1300 microwire array $(10 \mathrm{~mm}$ array diameter, $18 \mu \mathrm{m}$ wire diameter, $200 \mu \mathrm{m}$ spacing, $1 \mathrm{~mm}$ length), the largest microwire array 
implanted into the rodent cortex to date. The array was implanted into the somatosensory and prefrontal regions of the rat cortex for ease of insertion of the large array due to these areas being on a flatter part of the rat cortex and requiring minimal rotational manipulation of the headstage for insertion. Together, this allowed for successful array insertion normal to the surface of the brain. Using this array, we were able to isolate 791 single units (Figure 6A,C). Mean +/- SEM peak-to-peak amplitude, noise and SNR values obtained across all units were $130 \mu \mathrm{V}+/-59.7$, $10 \mu \mathrm{V}+/-2.2,8.9+/-1.9$ respectively (Supplementary Figure S1). Therefore, high fidelity neural activity can be recorded with the Argo recording system and associated microwire arrays along with a high unit count.

In addition to baseline recordings of spontaneous activity, pink noise auditory stimuli were presented to evoke somatosensory responses. Auditory stimuli were chosen in lieu of other sensory stimuli to avoid artifacts typically introduced by more direct somatosensory stimulation paradigms such as physical manipulation of the animal. We observed single units from somatosensory cortex that responded to auditory stimuli at an expected latency of $200 \mathrm{~ms}$ (Maruyama and Komai, 2018), further confirming neural activity (Figure 6D).

The rat cortex typically does not favor insertion of high density electrodes due to the brain size and high possibility of vascular insult (He et al., no date; Kozai et al., 2010; Blinder et al., 2013) resulting in damage to neural tissue. However, we were able to obtain high fidelity recording with high unit count (Figure 6A,B) owing to our microwire design (electro-sharpening) and ease of insertion into the rat cortex. 


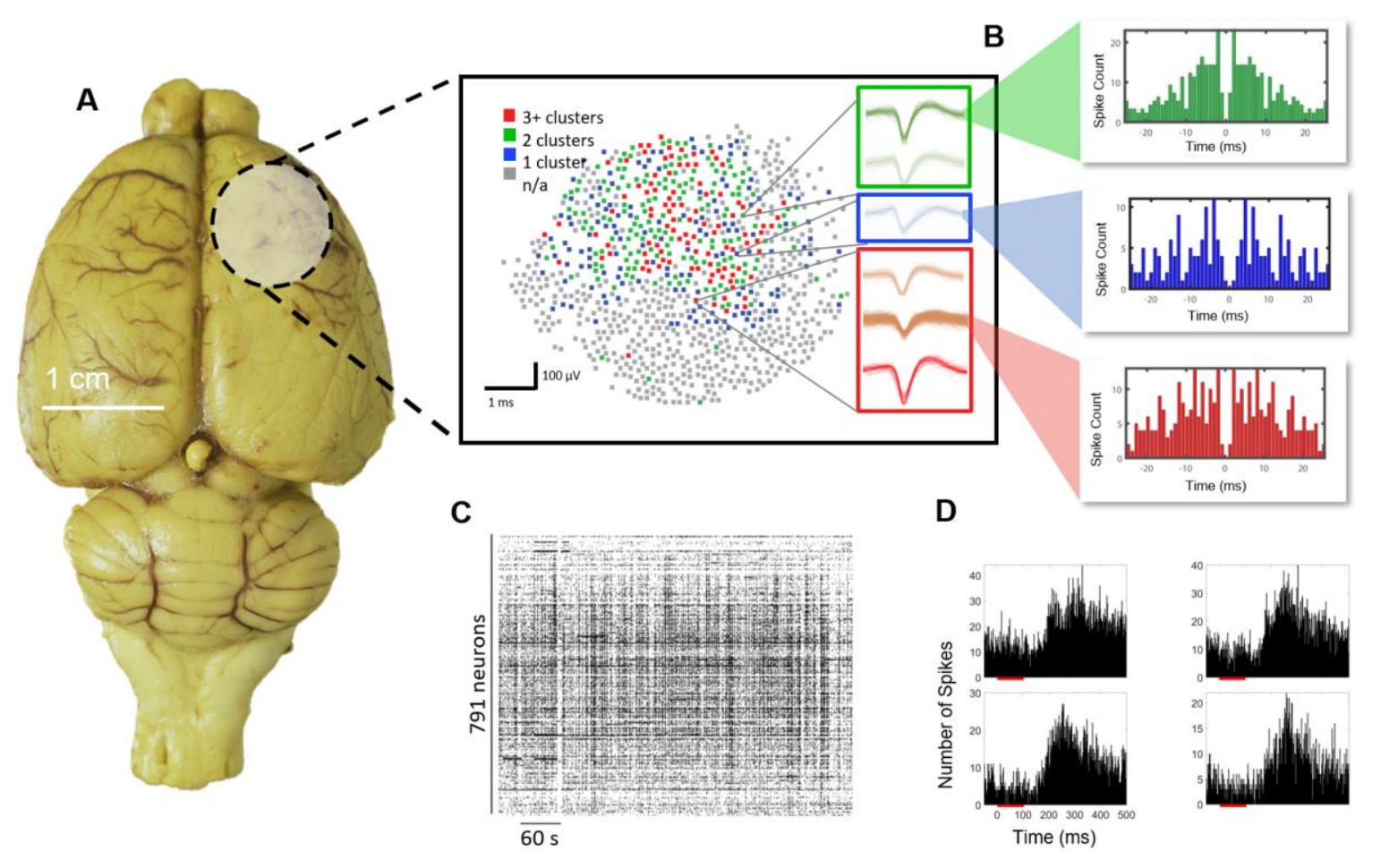

Figure 6. Rat cortex spike recordings. (A) Distribution of units found over the implantation of 1300 microwires in the rat cortex. The circular shaded region shows the location of the craniotomy and the brain region that was implanted, and the map shows channels with $0-3+$ spike clusters. (B) Example autocorrelogram from specific highlighted units, showing the emergence of well isolated single units. (C) A total of 791 single units as shown by the spike raster were found in the recording with good temporal dynamics associated with neuronal firing under anesthesia. (D) Example post-stimulus time histograms responses of single units in the somatosensory cortex to auditory pink noise stimuli (red bar). A latency of around $200 \mathrm{~ms}$ is observed, as expected from somatosensory neurons responding to auditory stimuli (Maruyama and Komai, 2018).

\section{Sheep Surface Recordings}

While the rat cortex was ideal to validate the Argo system's ability to record neural action potentials, the size of the microwire array was limited by the small size of the rodent brain. As a result, recordings would always be limited to 1000-2000 electrodes, substantially smaller than the maximum channel count of the Argo system. We therefore developed microwire arrays for surface LFP recordings with over 30,000 connected channels in order to evaluate surface potentials from sheep cortex $(n=2)$. The $\sim 30,000$ channel limit was the result of a tradeoff 
between electrode spacing, ideal microwire diameter and recording site area to allow for the acquisition of surface LFP at an appropriate spatial resolution.

The microwire arrays used in these recordings had a pitch of $60 \mu \mathrm{m}$, with an overall array size of $12 \mathrm{~mm} \times 12 \mathrm{~mm}$. We targeted the array over the auditory cortex (centering at $25 \mathrm{~mm}$ lateral and $5 \mathrm{~mm}$ anterior of bregma). LFPs were recorded at the surface of the cortex in response to pink noise (Figure 7) as well as pure tones (Figure 8). To quantify differences in evoked responses to pink noise compared to baseline activity, we first calculated the $\triangle \mathrm{RMS}$ for each channel in the $2 \mathrm{~Hz}$ to $400 \mathrm{~Hz}$ band (Figure 8A). As an initial measure of activation, we considered channels to be responsive if the evoked activity was at least $10 \mu \mathrm{V}$ RS higher than baseline, which is twice the determined noise floor. In one sheep, 21,963 channels of the 30,146 total channels $(73 \%)$ showed an increase in power above threshold during the pink noise stimulus, with $16,412(54 \%)$ of those channels' reponses being statistically significant. (Multiple comparisons nonparametric permutation test, $p<0.05$ ) (Nichols and Holmes, 2002). In a second sheep, we found that 17,233 of 31,239 total channels $(55 \%)$ showed increased power, with $12,590(40 \%)$ channels having a statistically significant response.

When presenting pure tone stimuli, we also observed frequency-specific responses (Figure 8), where 22,206 of $31,239(71 \%)$ channels had at least $10 \mu \mathrm{V}$ RMS activity above baseline and were significantly responsive to at least 2 tones (i.e., a minimum bandwidth of 0.6 octaves) ( $p<0.05$ Wilcoxon Rank Sum test, with Bonferroni correction). We then determined the BF for each channel by ranking the level of response to each frequency played. The BF for each channel was used to estimate the tonotopic organization across the cortex (Figure 8B). This was replicated in another experiment, where 26,054 of $30,146(86 \%)$ channels were responsive, with a similar tonotopy across the cortex (Supplementary Figure S2). 
A
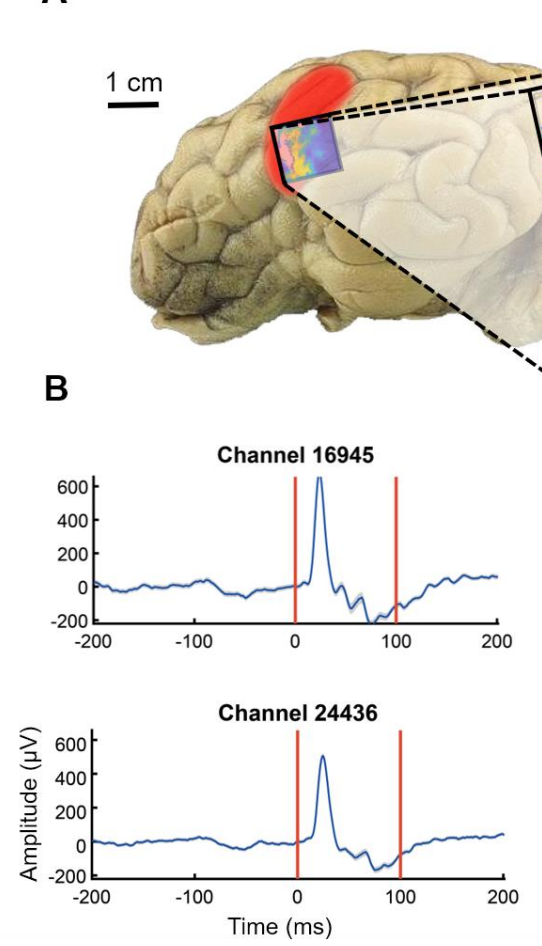

C

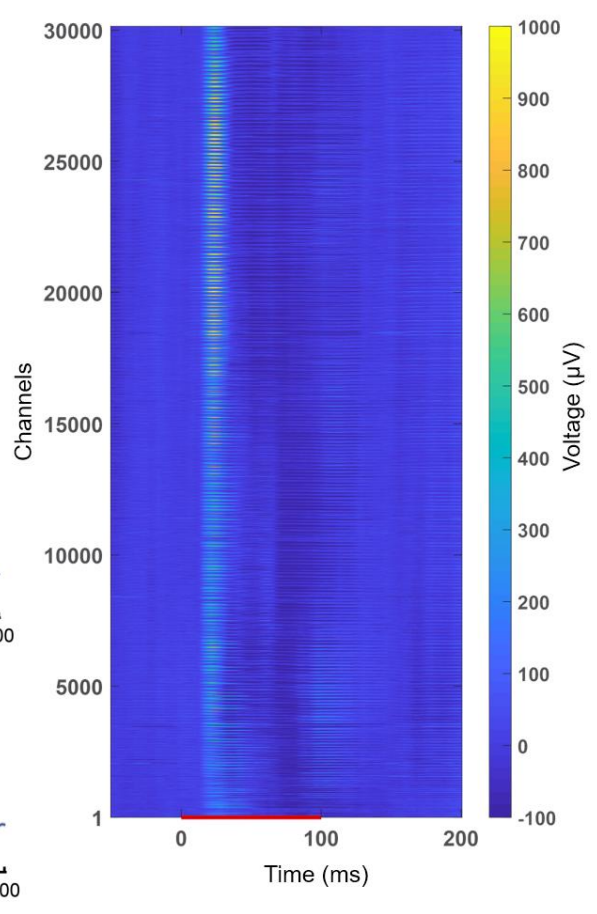

Figure 7. Pink noise-evoked potentials across the 30,000-channel microwire array from the sheep auditory cortex (red shaded area, described in (Gierthmuehlen et al., 2014)). (A) Location of the array (blue shaded boundary) for the auditory cortex surface recording and a map of $\Delta \mathrm{RMS}$ (i.e., response RMS minus baseline RMS) for individual channels in response to pink noise. With the $\Delta \mathrm{RMS}$ of at least $10 \mu \mathrm{V}, 21,963$ of these channels were responders to pink noise, $\mathrm{A}$ (anterior) and $D$ (dorsal) for the channel location in the array. (B) Evoked potentials (mean +/- SEM) from representative responding electrodes. (C) Trial-averaged evoked potentials of all 30,146 channels in response to pink noise. The red line denotes when the stimulus was presented. Channels were ordered spatially by the pixel location. 
A

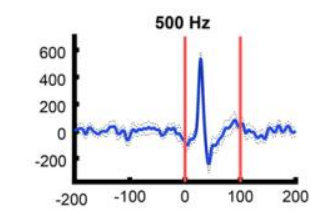

II
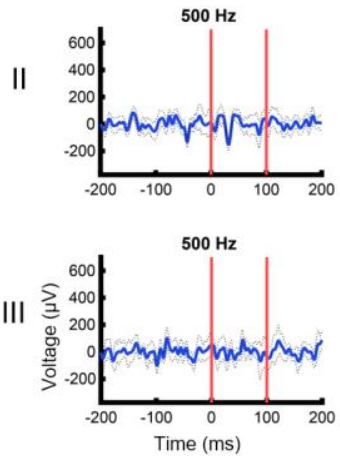
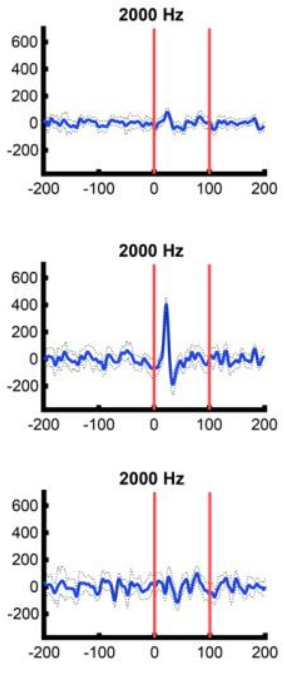
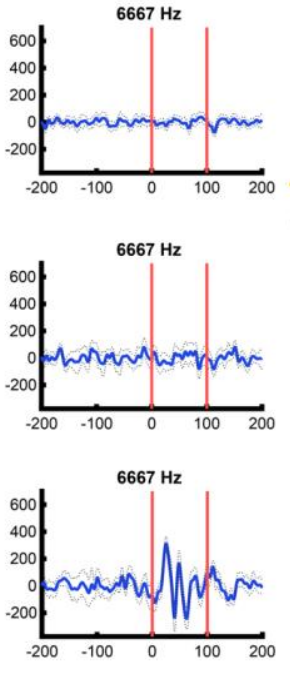

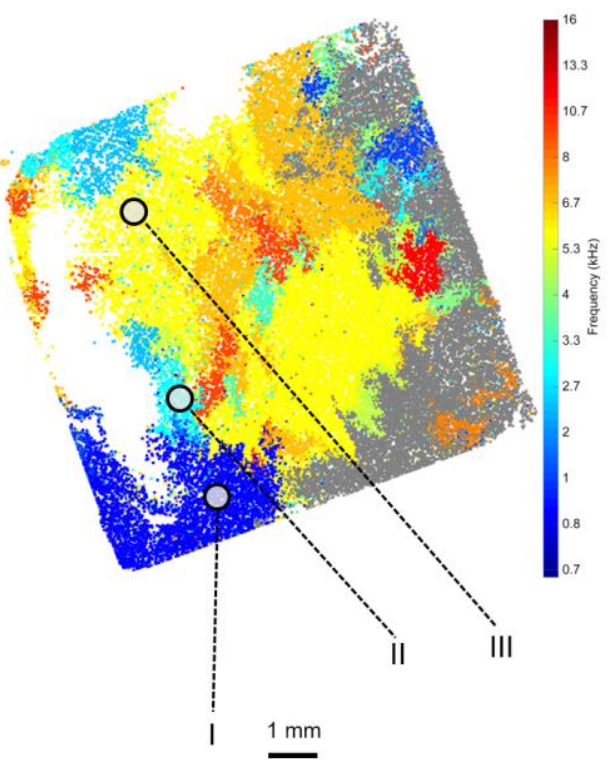

Figure 8. Frequency-specific responses with emerging tonotopic organization for the sheep auditory cortex. (A) Evoked potentials from electrodes across the microwire array demarcated from (I-III) are characteristic of frequency specific responses. (B) Array map with electrodes color coded for BF. Non-significantly responding electrodes shown in gray (Wilcoxon rank-sum, $p<0.05$, Bonferroni corrected). 


\section{Discussion:}

Here we have described and characterized a neural recording system based on microwire electrodes compressively and reversibly bonded to a custom-designed CMOS amplifier array. We have demonstrated that these recording devices provide large gain and a low noise floor and can scale to a very large number of recording sites with optimal gain and noise distributions. This makes such devices suitable for massively parallel electrical recordings of spikes and LFP in animal cortex.

To validate this recording capability, we have demonstrated the largest microwire electrode array-based recordings in both rat and large animal cortex to date. We show the ability to record from over 30,000 channels simultaneously at full acquisition rates $(32 \mathrm{kHz})$ in the sheep auditory cortex with good responses to auditory stimuli. Further, we show the ability to record high fidelity spikes in the rat cortex with our microwire based technology. Both results highlight and validate the capability to simultaneously record large channel count neural data at acquisition rates of $32 \mathrm{kHz}$ with the Argo system.

\section{Sheep Neural Recordings Demonstrate 30,000 channels of simultaneous acquisition}

From the sheep cortex, we recorded surface LFP on over 30,000 channels with a $12 \mathrm{~mm}$ $\times 12 \mathrm{~mm}$ array, which is the largest neural recording in a large animal to date. Other studies from $\mu E C o G$ in large animals and humans range from 16-294 channels (Chiang et al., 2020), therefore our system provides an order of magnitude increase in channel count. We performed surface LFP recordings using a high-density electrode array to mitigate the risk of damage to neurons that could have occurred upon insertion of a high-density penetrating array, resulting in poor recording quality.

To the best of our knowledge, this is also the first in vivo demonstration of evoked responses to auditory stimuli in sheep. The auditory cortex areas identified here were similar to those previously reported with histological tracing experiments in sheep (Michaloudi et al., 1986; 
Gierthmuehlen et al., 2014), which reported the auditory cortex to be approximately $1 \mathrm{~cm} \times 2 \mathrm{~cm}$. Responses to auditory stimuli typically had a latency of approximately $20 \mathrm{~ms}$ after stimulus onset, which is expected with surface LFP recordings from other relevant animal models (Kajikawa and Schroeder, 2011; Escabí et al., 2014; Trumpis et al., 2017). We did observe slightly different responses to tones across the microwire array between experiments, with one experiment revealing more channels with higher BFs. This is likely due to small differences in placement, especially since the array could span approximately half of the auditory cortex, and individual animal variation. Moreover, this tonotopy and sound-evoked responses were evident despite the acoustically noisy environment (i.e., an acute operating room setting rather than the traditional sound booth environment used in traditional auditory experiments), which could have led to increased variability in responses. However, even with this variation, we were able to find emerging tonotopy in the sheep auditory cortex.

\section{Rat Neural Recordings Reveal High-fidelity Action Potentials}

We demonstrate the ability to record from 791 single units in the rat cortex. This is significantly more than other microwire technology that has been used to record from the rodent cortex, where the number of neurons ranges from 20-240 units in an acute setting (Guitchounts et al., 2013; Pfeiffer and Foster, 2013; Chung et al., 2019; Massey et al., 2019; Obaid et al., 2020, no date). We found the mean SNR across all channels to be close to 9, indicative of high fidelity recordings given that typical values using this metric define good SNR between 3 and 6 (Ward et al., 2009; Ludwig et al., 2011; Kozai et al., 2012; Sohal et al., 2014; Xu et al., 2018). Here we demonstrate a system that is capable of producing low noise and high captured signal amplitude from single units. This is enabled in large part by the combination of tunable high pass and low pass filters that allow us to set the input bandwidth to reject both low and high frequency noise and remove aliasing effects. 
Furthermore, the design of the microwires enables good performance for neural recordings. Specifically, our microwire electrodes have tip based recording sites, which are known to produce better recording quality compared to those sites that are along the length of the shaft, like some multi-depth silicon probes (Kipke et al., 2003; Jun et al., 2017). The electrosharpening of the tips also allows for ease of high-density array insertion into the brain (Obaid et al., 2020, no date) as has been demonstrated by our neural recordings, where we can successfully insert arrays into the rat cortex.

\section{Microwire-CMOS devices as Next Generation Brain-Computer Interfaces}

Microwire-CMOS devices combine the robustness and longevity of traditional microwire electrodes with the advantages of active CMOS probes. Specifically, the design overcomes three of the greatest drawbacks of using microwire electrode arrays for neural recording. 1) By amplifying close to the signal source, the design reduces both input capacitance and noise pickup. 2) By providing on-chip multiplexing, the design allows for a much smaller number of lead wires and connectors than electrode sites. 3) Bonding pre-assembled arrays of microwires onto a CMOS sensor array provides a much simpler method of connecting the microwires than the traditional hand-wiring approach.

Here we demonstrate that microwire-CMOS is indeed scalable to tens of thousands of channels, but the current system is limited to acute, head-fixed preparations due to the size of the electronic components that lie downstream of the CMOS amplifier array. The clear next step is to develop a new ASIC and downstream architecture that are compatible with a floating microwire array configuration. Such a device would have a form factor similar to the Utah array but allow for greater electrode density, reduced tissue damage and insertion forces owing to smaller electrode diameter (Patel et al., 2015; Obaid et al., 2020, no date), and reduced interface cable thickness due to on-chip multiplexing of channels. Development of such a device is already underway. 


\section{Acknowledgments}

This work was funded by a Small Business Innovative Research grant from the National Institute of Health Brain Initiative (5R43MH110287-02) and by the Defense Advanced Research Projects Agency's Neural Engineering System Design program (N66001-17-C-4005). The authors would like to thank Prof. Andreas Schaefer for feedback and discussions, and Dr. Marike Zwienenberg, Linda Talken, and Amy Lesneski (UC Davis) for help in developing sheep surgical protocols. Competing interests: K.S., A.A.K., A.P.S., T.M.S, Y.N., A.T., P.O., C.L., D.P., K.N., K.M.B., S.S., M.S.H., B.K., M-E.S.H., R.J.E., I.M., D.F., A.M.S., V.G., Y.K., M.S., H.S.S., M.R.A. are current or former compensated employees or consultants of Paradromics, Inc., a brain-computer interface company. P.G., A.B-F, S.V., A.V.K, B.L., B.D. are compensated employees or consultants of Caeleste, CVBA, a circuit design company. 


\section{References}

Acker, L. et al. (2016) 'FEF inactivation with improved optogenetic methods', Proceedings of the National Academy of Sciences of the United States of America, 113(46), pp. E7297-E7306.

Bartels, J. et al. (2008) 'Neurotrophic electrode: method of assembly and implantation into human motor speech cortex', Journal of neuroscience methods, 174(2), pp. 168-176.

Blinder, P. et al. (2013) 'The cortical angiome: an interconnected vascular network with noncolumnar patterns of blood flow', Nature neuroscience, 16(7), pp. 889-897.

Cash, S. S. and Hochberg, L. R. (2015) 'The emergence of single neurons in clinical neurology', Neuron, 86(1), pp. 79-91.

Ceyssens, F. and Puers, R. (2015) 'Insulation lifetime improvement of polyimide thin film neural implants', Journal of neural engineering, 12(5), p. 054001.

Chang, W.-T. et al. (2012) 'Method of electrochemical etching of tungsten tips with controllable profiles', The Review of scientific instruments, 83(8), p. 083704.

Chen, T.-W. et al. (2013) 'Ultrasensitive fluorescent proteins for imaging neuronal activity', Nature, 499(7458), pp. 295-300.

Chiang, C.-H. et al. (2020) 'A modular high-density $\mu$ ECoG system on macaque vIPFC for auditory cognitive decoding', Journal of neural engineering. doi: 10.1088/1741-2552/ab9986.

Chung, J. E. et al. (2019) 'High-Density, Long-Lasting, and Multi-region Electrophysiological Recordings Using Polymer Electrode Arrays', Neuron, 101(1), pp. 21-31.e5.

Cogan, S. F. (2008) 'Neural stimulation and recording electrodes', Annual review of biomedical engineering, 10, pp. 275-309.

Čvančara, P. et al. (2020) 'Stability of flexible thin-film metallization stimulation electrodes: analysis of explants after first-in-human study and improvement of in vivo performance', Journal of neural engineering. doi: 10.1088/1741-2552/ab9a9a.

Dana, H. et al. (2019) 'High-performance calcium sensors for imaging activity in neuronal populations and microcompartments', Nature methods, 16(7), pp. 649-657.

Escabí, M. A. et al. (2014) 'A high-density, high-channel count, multiplexed $\mu$ ECoG array for auditory-cortex recordings', Journal of neurophysiology, 112(6), pp. 1566-1583.

Flesher, S. N. et al. (2016) 'Intracortical microstimulation of human somatosensory cortex', Science translational medicine, 8(361), p. 361ra141.

Geddes, L. A. and Roeder, R. (2003) 'Criteria for the selection of materials for implanted electrodes', Annals of biomedical engineering, 31(7), pp. 879-890.

Ghanbari, Y., Papamichalis, P. and Spence, L. (2009) 'Robustness of neural spike sorting to sampling rate and quantization bit depth', 2009 16th International Conference on Digital Signal 
Processing. doi: 10.1109/icdsp.2009.5201163.

Gierthmuehlen, M. et al. (2014) 'Mapping of sheep sensory cortex with a novel microelectrocorticography grid', The Journal of comparative neurology, 522(16), pp. 35903608.

Guitchounts, G. et al. (2013) 'A carbon-fiber electrode array for long-term neural recording', Journal of neural engineering, 10(4), p. 046016.

He, F. et al. (no date) 'Multimodal mapping of neural activity and cerebral blood flow reveals long-lasting neurovascular dissociations after small-scale strokes'. doi:

10.1101/2020.03.04.977322.

Hochbaum, D. R. et al. (2014) 'All-optical electrophysiology in mammalian neurons using engineered microbial rhodopsins', Nature methods, 11(8), pp. 825-833.

Hochberg, L. R. et al. (2012) 'Reach and grasp by people with tetraplegia using a neurally controlled robotic arm', Nature, 485(7398), pp. 372-375.

Hubel, D. H. (1959) 'Single unit activity in striate cortex of unrestrained cats', The Journal of physiology, 147, pp. 226-238.

Hubel, D. H. and Wiesel, T. N. (1959) 'Receptive fields of single neurones in the cat's striate cortex', The Journal of Physiology, pp. 574-591. doi: 10.1113/jphysiol.1959.sp006308.

Hughes, C. L. et al. (no date) 'Neural stimulation and recording performance in human somatosensory cortex over 1500 days'. doi: 10.1101/2020.01.21.20018341.

Hyvärinen, A. and Oja, E. (2000) 'Independent component analysis: algorithms and applications', Neural networks: the official journal of the International Neural Network Society, 13(4-5), pp. 411-430.

Jackson, A. and Fetz, E. E. (2007) 'Compact Movable Microwire Array for Long-Term Chronic Unit Recording in Cerebral Cortex of Primates', Journal of Neurophysiology, pp. 3109-3118. doi: 10.1152/jn.00569.2007.

Jun, J. J. et al. (2017) 'Fully integrated silicon probes for high-density recording of neural activity', Nature, 551(7679), pp. 232-236.

Kajikawa, Y. and Schroeder, C. E. (2011) 'How local is the local field potential?', Neuron, 72(5), pp. 847-858.

Khan, A. et al. (2018) 'Data pipeline for 65,536 channels of extracellular unit recordings', 40th Annual International Conference of the IEEE Engineering in Medicine and Biology Society (EMBC), Honolulu, HI, 2018, WeAT17.3.

Kipke, D. R. et al. (2003) 'Silicon-substrate intracortical microelectrode arrays for long-term recording of neuronal spike activity in cerebral cortex', IEEE transactions on neural systems and rehabilitation engineering: a publication of the IEEE Engineering in Medicine and Biology Society, 11(2), pp. 151-155. 
Kollo, M. et al. (no date) 'CHIME: CMOS-hosted in-vivo microelectrodes for massively scalable neuronal recordings'. doi: 10.1101/570069.

Kozai, T. D. Y. et al. (2010) 'Reduction of neurovascular damage resulting from microelectrode insertion into the cerebral cortex using in vivo two-photon mapping', Journal of neural engineering, 7(4), p. 046011.

Kozai, T. D. Y. et al. (2012) 'Ultrasmall implantable composite microelectrodes with bioactive surfaces for chronic neural interfaces', Nature materials, 11(12), pp. 1065-1073.

Kozai, T. D. Y. et al. (2015) 'Mechanical failure modes of chronically implanted planar siliconbased neural probes for laminar recording', Biomaterials, 37, pp. 25-39.

Kozai, T. D. Y. and Vazquez, A. L. (2015) 'Photoelectric artefact from optogenetics and imaging on microelectrodes and bioelectronics: New Challenges and Opportunities', Journal of materials chemistry. B, Materials for biology and medicine, 3(25), pp. 4965-4978.

Lewicki, M. S. (1998) 'A review of methods for spike sorting: the detection and classification of neural action potentials', Network: Computation in Neural Systems, pp. R53-R78. doi: 10.1088/0954-898x_9_4_001.

Lubin, J. A., Strebe, J. K. and Kuo, J. S. (2017) 'Intracortical Microstimulation of Human Somatosensory Cortex Reproduces Touch in Spinal Cord Injury Patient', Neurosurgery, 80(5), pp. N29-N30.

Ludwig, K. A. et al. (2009) 'Using a common average reference to improve cortical neuron recordings from microelectrode arrays', Journal of neurophysiology, 101(3), pp. 1679-1689.

Ludwig, K. A. et al. (2011) 'Poly(3,4-ethylenedioxythiophene) (PEDOT) polymer coatings facilitate smaller neural recording electrodes', Journal of neural engineering, 8(1), p. 014001.

Maruyama, A. T. and Komai, S. (2018) 'Auditory-induced response in the primary sensory cortex of rodents', PloS one, 13(12), p. e0209266.

Massey, T. L. et al. (2019) 'A high-density carbon fiber neural recording array technology', Journal of neural engineering, 16(1), p. 016024.

McMahon, D. B. T. et al. (2014) 'One month in the life of a neuron: longitudinal single-unit electrophysiology in the monkey visual system', Journal of neurophysiology, 112(7), pp. 17481762.

Michaloudi, H. et al. (1986) 'Thalamic projections to the posterior sylvian and posterior ectosylvian gyri of the sheep brain, revealed with the retrograde transport of horseradish peroxidase', Anatomy and embryology, 175(1), pp. 77-90.

Misra, A. et al. (2014) 'Methods for implantation of micro-wire bundles and optimization of single/multi-unit recordings from human mesial temporal lobe', Journal of Neural Engineering, p. 026013. doi: 10.1088/1741-2560/11/2/026013.

Musk, E. and Neuralink (2019) 'An Integrated Brain-Machine Interface Platform With Thousands of Channels', Journal of medical Internet research, 21(10), p. e16194. 
Musselman, I. H. and Russell, P. E. (1990) 'Platinum/iridium tips with controlled geometry for scanning tunneling microscopy', Journal of vacuum science \& technology. A, Vacuum, surfaces, and films: an official journal of the American Vacuum Society. American Vacuum Society, 8(4), pp. 3558-3562.

Nichols, T. E. and Holmes, A. P. (2002) 'Nonparametric permutation tests for functional neuroimaging: a primer with examples', Human brain mapping, 15(1), pp. 1-25.

Nicolelis, M. A. L. et al. (2003) 'Chronic, multisite, multielectrode recordings in macaque monkeys', Proceedings of the National Academy of Sciences of the United States of America, 100(19), pp. 11041-11046.

Nordhausen et al. (1992) 'A Method For Acute Cerebral Cortex Recordings Using The Utah Intracortical Electrode Array', Proceedings of the Annual International Conference of the IEEE Engineering in Medicine and Biology Society. doi: 10.1109/iembs.1992.592715.

Obaid, A. et al. (2020) 'Massively Parallel Microwire Arrays Integrated with CMOS chips for Neural Recording', Science Advances. doi: 10.1126/sciadv.aay2789.

Obaid, A. et al. (no date) 'Ultra-sensitive measurement of brain penetration with microscale probes for brain machine interface considerations'. doi: 10.1101/454520.

Pandarinath, C. et al. (2017) 'High performance communication by people with paralysis using an intracortical brain-computer interface', eLife, 6. doi: 10.7554/eLife.18554.

Patel, P. R. et al. (2015) 'Insertion of linear $8.4 \mu \mathrm{m}$ diameter 16 channel carbon fiber electrode arrays for single unit recordings', Journal of neural engineering, 12(4), p. 046009.

Pfeiffer, B. E. and Foster, D. J. (2013) 'Hippocampal place-cell sequences depict future paths to remembered goals', Nature, pp. 74-79. doi: 10.1038/nature12112.

Piatkevich, K. D. et al. (2018) 'A robotic multidimensional directed evolution approach applied to fluorescent voltage reporters', Nature chemical biology, 14(4), pp. 352-360.

Quiroga, R. Q., Nadasdy, Z. and Ben-Shaul, Y. (2004) 'Unsupervised spike detection and sorting with wavelets and superparamagnetic clustering', Neural computation, 16(8), pp. 16611687.

Rossant, C. et al. (2016) 'Spike sorting for large, dense electrode arrays', Nature neuroscience, 19(4), pp. 634-641.

Rousche, P. J. and Normann, R. A. (1998) 'Chronic recording capability of the Utah Intracortical Electrode Array in cat sensory cortex', Journal of neuroscience methods, 82(1), pp. 1-15.

Schwarz, D. A. et al. (2014) 'Chronic, wireless recordings of large-scale brain activity in freely moving rhesus monkeys', Nature methods, 11(6), pp. 670-676.

Sohal, H. S. et al. (2014) 'The sinusoidal probe: a new approach to improve electrode longevity', Frontiers in neuroengineering, 7, p. 10.

Stevenson, I. H. and Kording, K. P. (2011) 'How advances in neural recording affect data 
analysis', Nature Neuroscience, pp. 139-142. doi: 10.1038/nn.2731.

Stringer, C. et al. (2019) 'Spontaneous behaviors drive multidimensional, brainwide activity', Science, 364(6437), p. 255.

Strumwasser, F. (1958) 'Long-Term Recording from Single Neurons in Brain of Unrestrained Mammals', Science, pp. 469-470. doi: 10.1126/science.127.3296.469.

Truccolo, W. et al. (2011) 'Single-neuron dynamics in human focal epilepsy', Nature neuroscience, 14(5), pp. 635-641.

Trumpis, M. et al. (2017) 'A low-cost, scalable, current-sensing digital headstage for high channel count $\mu \mathrm{ECoG}$ ', Journal of neural engineering, 14(2), p. 026009.

Ward, M. P. et al. (2009) 'Toward a comparison of microelectrodes for acute and chronic recordings', Brain research, 1282, pp. 183-200.

Willett, F. R. et al. (no date) 'High-performance brain-to-text communication via imagined handwriting'. doi: 10.1101/2020.07.01.183384.

Xie, X. et al. (2014) 'Long-term reliability of Al2O3 and Parylene C bilayer encapsulated Utah electrode array based neural interfaces for chronic implantation', Journal of neural engineering, $11(2)$, p. 026016.

$\mathrm{Xu}, \mathrm{H}$. et al. (2018) 'Acute in vivo testing of a conformal polymer microelectrode array for multiregion hippocampal recordings', Journal of neural engineering, 15(1), p. 016017. 